\title{
2016 Acknowledgments
}

Psychology of Popular Media Culture is very fortunate to have a wide pool of talented reviewers to complement the editorial board. The Editors would like to thank the following scholars for contributing their expertise and energy.

Paul Adachi

Nelson Adie

Phyllis Anastasio

Craig Anderson

Daniel R. Anderson

Shayne Aquino

Diane Ashe

Eleni-Marina Ashikali

Jennifer Stevens Aubrey

Robert A. Backer

Suzanne Baker

Jaime Banks

Martin S. Banks

Tom Baranowski

Baptiste Barbot

Bruce Bartholow

Anne Bartsch

Anamitra Basu

Mark Batey

Anthony Martin Bean

Elizabeth Behm-

Morawitz

Rebecca Bigler

Joel Billieux

Max Birk

Fran Blumberg

Paul Booth

Kelly Boudreau

Andy Boyan

Nevfel Boz

Loretta L. C. Brady

Matthias Brand

Nina Brathwaite

Johannes Breuer

Britney G. Brinkman

Jeanne Brockmyer

Francette Broekman

Colton Abraham Brown

Lyn Brown

Steven Brown

William J. Brown

Jacob M. Burmeister

Rick Busselle
Jerel Calzo

Keith Campbell

Kelly Campbell

Chase D. Cartwright

Reuben Mikhael

Castagno

Kara Chan

Makana Chock

Sally Chung

Vincent Cicchirillo

Annabel Cohen

M. Nicole Coleman

Emily Collins

Theodore V. Cooper

Tressie M. Cottom

Mark Coulson

Sarah Coyne

Cynthia A. Crawford

Donna Cross

Christina C. Crowe

Laura Crysel

Elizabeth A. Daniels

Leslie Alison Davis

Whitney DeCamp

Lauren J. DeCarvalho

Edward Deci

Renee Dennison

Jaye L. Derrick

Serge Desmarais

Grant James Devilly

C. Nathan DeWall

Anne Dickmeis

Don Diefenbach

Kelly Dillon

Edward Downs

Athena A. Drewes

Kristin Drogos

Michelle Drouin

Aaron Drummond

Mark Duffett

Robert Andrew Dunn

Eva Duven

Carla Earhart
Allison Eden

Christian Edwards

Morgan E. Ellithorpe

Malte Elson

Elisabeth Engelberg

Mindy Engle-Friedman

Sarah Erickson

Rocio FernandezBallesteros

Ruth Festi

Adam R. Fisher

Brad Forenza

Marie J. C. Forgeard

Jesse Fox

R. Chris Fraley

Carly Friedman

Susan Hatters Friedman

Yuki Fujioka

Chris Fullwood

Adrian Furnham

Amber M. Gaffney

Antonis Gardikiotis

Kathrin Gerling

Sarah Gervais

Bryan Gibson

David Giles

Meghan M. Gillen

Janet Gillett

Thalia R. Goldstein

Gordon Goodman

John Goodwin

Amelia Gosal

Jessica Gotthold

Abbie Grace

Garo Green

Melanie Green

Melinda A. Green

David Greenberg

Dara Greenwood

Rosanna E. Guadagno

Angie Guan

Andrea Guazzini

Georges-Claude Guilbert 
Gert Martin Hald

Alice Hall

Erika Hall

Michael David Hanus

Christian Happ

David Hargreaves

C. Lee Harrington

Kristen Harrison

Youssef Hasan

Linda Heath

Veronica Hefner

Amir Hetsroni

Joseph Hilgard

August John Hoffman

Cynthia Hoffner

Emily Horowitz

Jim Houran

$\mathrm{Mu} \mathrm{Hu}$

L. Rowell Huesmann

Margret Hunter

Stacey Hust

James D. Ivory

Sophie Helga Janicke

Michael Jenkins-

Guarnieri

Robert Jensen

Sven Jöckel

Benjamin K. Johnson

Brian D. Johnson

Peter Karl Jonason

Eric E Jones

Hansika Kapoor

Jen Katz-Buonincontro

Geoff Kaufman

Linda K. Kaye

Kerk F. Kee

Pelin Kesebir

Selin Kesebir

Allison Kiefner-

Burmeister

Janna Kim
Alexandra C. Kirsch

Christoph Klimmt

Rachel Kowert

Nicole Krämer

Linda Kreger

Daria Kuss

Kyounghee Hazel Kwon

Marianne LaFrance

Travis Langley

Michael Laroche

Samuel Leistedt

Lisa Libby

Anthony Limperos

Jennifer Linder

Deborah Linebarger

Julia R. Lippman

Adam Lobel

Meghan R. Longacre

Paul Lopes

Ying Lu

Robert Benjamin Lull

Sara Magee

Chad Mahood

Julia A. Maier

John Maltby

Raymond Mar

Patrick Markey

Lynn McCutcheon

Brandon T. McDaniel

Andrea McDonnell

D. Cricket Meehan

André Melzer

Aaron Mendelovitz

Brandon Miller

Jyoti Mishra

Beth Morling

Todd Morrison

Thomas Mößle

Dirk Oliver Mügge

Shane Murphy

Nicole Muscanell

Taryn Myers
Athi Myint-U

Jessica Gall Myrick

Matt Newman

Benjamin Wai-ming Ng

Jeeyun Oh

Shigehiro Oishi

Oluyinka Ojedokun

Julian Oldmeadow

Mary Beth Oliver

Cheryl Olson

Lucia O'Sullivan

Wei Peng

Kylie Peppler

Robert Perl

Courtney Plante

Jonathan A. Plucker

Halley Pontes

Andrew Przybylski

Thorsten Quandt

Sally Quinn

Arthur Raney

Apara Ranjan

Daniel Re

Jenny Read

Leonard Reinecke

Peter Jason Rentfrow

Lina Ricciardelli

Karyn Riddle

Diana Rieger

Scott Rigby

Ian Rivers

Daniel Dan Romer

Thomas William Ross

Dana Ruggiero

Nicholas Rule

Kevin Runions

Pamela Rutledge

Richard Ryan 
Goal Auzeen Saedi

Valorie N. Salimpoor

Meghan Sanders

Don Saucier

Karen Saules

Paddy Scannel

E. Glenn Schellenberg

Tatiana Orozco

Schember

Judith Schlesinger

Michael Schmierbach

Anna Schnauber

Christina Schumann

E. Scott

Suzanne Scott

Daniel M. Shafer

Chirag Shah

Brian Carey Sims

Dorothy Singer

Jennie K. Singer

Marko Skoric

Rebekah Smart

Jeffrey Smith

Glenn Sparks
Shirley Steinberg

Gayle Stever

Deryn Strange

Ray Surette

Rachael Talpash

Chee Seng Tan

Yi-Yuan Tang

Christa L. Taylor

Laramie D. Taylor

Morgan James Tear

Joel Thompson

Deborah Tolman

Michael Toohey

Raphael Travis

Jessica Troilo

Mina Tsay-Vogel

Konstantin Tskhay

Marion Underwood

Sonja Utz

Manford Van Dulmen

Jan Van Looy
John Velez

Lotte Vermeulen

Cynthia Vinney

Erin Alyssa Vogel

Scott Vrana

Anthony Wagner

Otto Wahl

Daniel Wann

Robert S. Weisskirch

Arielle White

Erin Whiteside

Jessica Williams

June Wilson

Jeffrey Wimmer

Karen Wohlwend

Julia Woolley

Michelle Wright

Jeffery Yen

Jason Young

Adam Zimmerman

Eileen Zurbriggen 